\title{
Fibroma of tendon sheath as a cause of carpal tunnel syndrome
}

\author{
Jaggi Rao BSc, Achilleas Thoma MD FRCSC FACS, Sam Salama MB FRCPC \\ Department of Surgery, Division of Plastic and Reconstructive Surgery, and Department of Pathology, \\ St Joseph's Hospital, McMaster University, Hamilton, Ontario
}

\begin{abstract}
J Rao, A Thoma, S Salama. Fibroma of tendon sheath as a cause of carpal tunnel syndrome. Can J Plast Surg 1997;5(3):176-178. This report describes a 28-year-old male with a fibrovascular tumour of the left midpalm, extending into the carpal tunnel. It compressed the median nerve, producing carpal tunnel syndrome. Upon carpal tunnel decompression and local excision, the symptoms were relieved. Histopathological study of the tumour revealed characteristics suggestive of fibroma of tendon sheath, an uncommon tumour of the hand and rare cause of carpal tunnel syndrome.
\end{abstract}

Key Words: Carpal tunnel syndrome, Fibroma of tendon sheath

\section{Fibrome de la gaine du tendon en cause dans le syndrome du tunnel carpien}

RÉSUMÉ : Ce rapport décrit le cas d'un homme de 28 ans atteint d'une tumeur fibrovasculaire au milieu de la paume gauche s'étendant vers le tunnel carpien. La tumeur comprimait le nerf médian entraînant un syndrome du tunnel carpien. Lors de la décompression du tunnel carpien et d'une excision locale, les symptômes ont été soulagés. L'examen histopathologique de la tumeur a révélé des caractéristiques évocatrices d'un fibrome de la gaine du tendon, une tumeur rare de la main et une cause rare du syndrome du tunnel carpien.

$\mathrm{I}_{\mathrm{b}}^{\mathrm{n}}$ 1949, Geschickter and Copeland (1) first described fibroma of tendon sheath (FTS) as a distinct entity, but it was not described further until 1979, when Chung and Enzinger (2) presented the clinical and pathological findings of 138 cases selected from the files of the Armed Forces Institute of Pathology. In all cases of FTS reported in literature, only six have been implicated as a cause of carpal tunnel syndrome (CTS). The fibroma described in this report is an example of this rare tumour; it led to the progression of CTS and demonstrated characteristics unique to FTS. Carpal tunnel release and local excision of the tumour promptly relieved the patient's symptoms.

\section{CASE PRESENTATION}

A 28-year-old Caucasian male presented with a complaint of cramping in the left hand, as well as numbness to the left middle and ring fingers. With minimal activity of the hand, there was swelling of the midpalm, and he became more symptomatic.

Correspondence and reprints: Dr Achilleas Thoma, 200 James Street South (Suite 208), Hamilton, Ontario L8P 3A9. Telephone 905-523-0019, fax 905-523-0229, e-mail athoma@fhs.mcmaster.ca
Seven months earlier, the patient underwent surgery to remove the tumour in a peripheral hospital. The tumour was adhered to the median nerve. The original surgeon, not feeling confident about the nature of the tumour, prudently abandoned the procedure. A biopsy was performed instead, which, although nondiagnostic, suggested a benign fibrovascular tumour.

The patient continued to suffer numbness in the median nerve distribution, particularly on the contiguous surfaces of the middle and ring fingers. Three months following the initial attempt at resection, he was forced to take leave from his occupation as a butcher because of midpalmar pain.

At the present examination, the patient had a palpable, soft tissue mass in the left palm, located at the distal portion of the carpal tunnel. The mass was tender to pressure and the provocative tests for CTS, including Tinel's and Phalen's tests, were positive. Accompanying this was paresthesia on both sides of the middle and ring fingers.

Consent was obtained for exploration and resection of the tumour with the strong possibility of sural nerve grafting. At surgery, an encapsulated whitish-grey firm tumour was identified which resembled a benign schwannoma (Figure 1). It extended from the hypothenar to the thenar eminence and filled the distal portion of the carpal tunnel. 


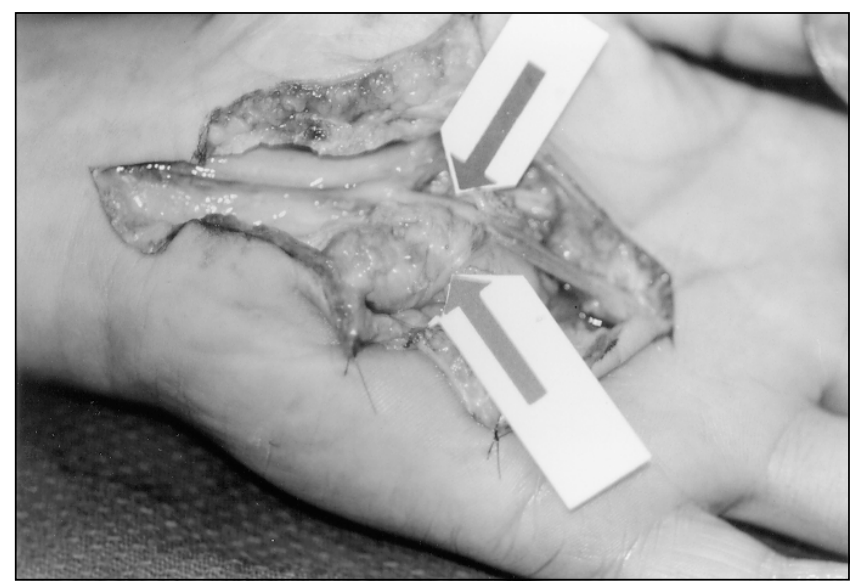

Figure 1) Intraoperative appearance of fibroma of tendon sheath. Inferior arrow points to distal extent of the tumour. A branch of the median nerve is stretched by the underlying tumour (superior arrow)

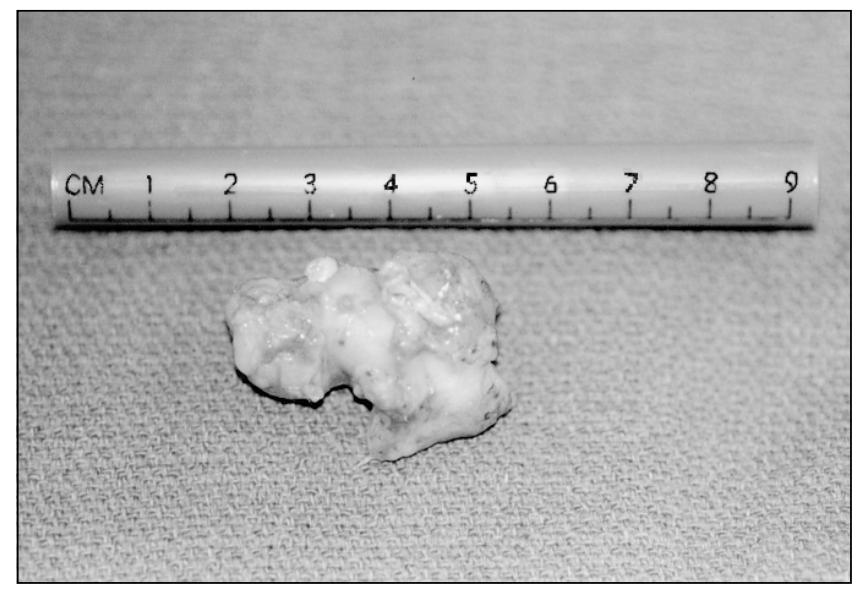

Figure 2) Gross appearance of removed tumour

Removal of the tumour required complete decompression of the carpal tunnel through a linear incision extending to the proximal wrist crease, and exposure of Guyon's canal to identify the ulnar artery and nerve.

The communicating branch of the ulnar and median nerves stretched over the capsule, and was identified and preserved. The common digital artery to the middle and ring fingers was encased by the tumour, and was therefore ligated proximally and distally, and sacrificed. All the digital nerves were preserved.

The motor branches to the lumbricals of the middle and ring fingers were involved in the tumour and were sacrificed (during surgery, these nerves were thought to be the origin of this presumptive schwannoma). The recurrent branch of the median nerve was teased off the capsule and preserved. The tenosynovial tissue was stripped off the flexer tendons in the carpal tunnel and excised en bloc with the tumour.

The excised tumour measured $3.6 \times 1.6 \times 0.9 \mathrm{~cm}$ and was firm, nodular, well circumscribed and grey-tan coloured, with a thin layer of adipose tissue (Figure 2). Microscopic examination revealed an encapsulated benign fibrovascular lesion consisting of spindle-shaped fibroblasts embedded

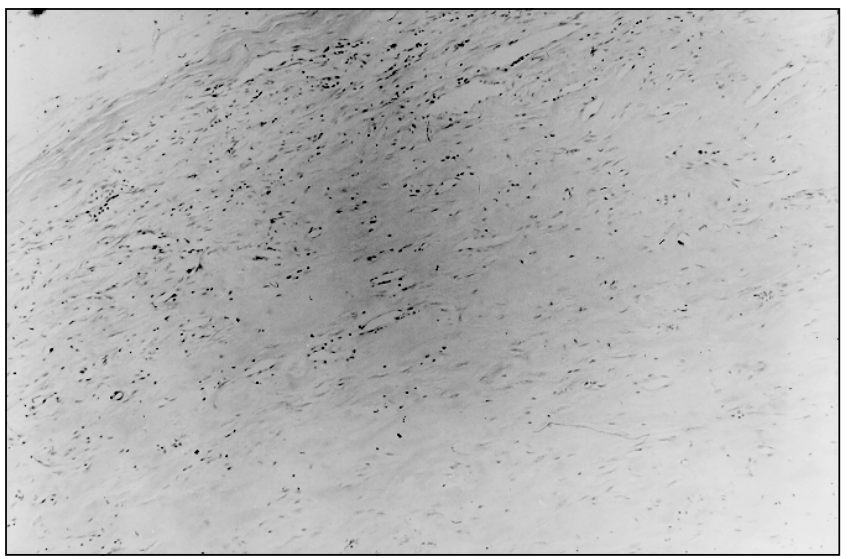

Figure 3) The tumour appears encapsulated and shows fibrocollagenous stroma. Notice the prominent vasculature with small blood vessels (hematoxylin and eosin $\mathrm{x} 100$ )

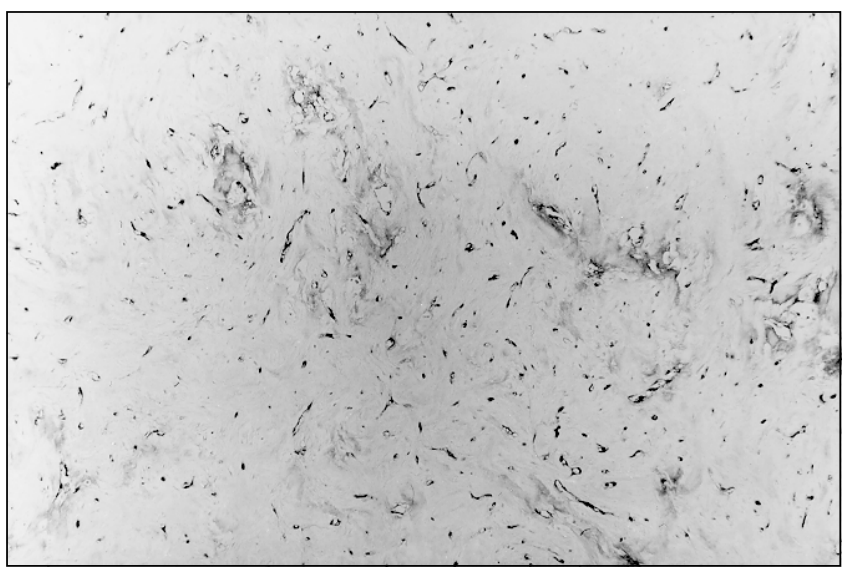

Figure 4) Section of the tumour stained with the factor VIII antibody, showing prominent vascularized stroma (peroxidase antiperoxidase 100 )

within dense fibrocoliagenous stroma (Figure 3). The stroma also exhibited vascular spaces, but no giant cells were noted (Figure 4). Few lymphocytes were seen scattered along the periphery of the tumour. No neural structures were seen around the tumour.

Postoperatively, the patient's symptoms disappeared, and he was able to return to work four weeks later.

\section{DISCUSSION}

Compression from benign tumours has been included in the differential diagnosis of CTS, and although documentation is rare, FTS is no exception. In the Chung and Enzinger study (2), two patients who had FTS in the hand showed symptoms of CTS, while another patient showed paresthesia. Sarma et al (3) reported an encapsulated FTS found during carpal tunnel release. This tumour was attached to the flexer digitorum profundus tendon of the middle finger and was compressing the median nerve enough to cause a complete loss of sensation along its distribution, as well as partial block of flexion of the third digit. One patient suffering from FTS in the midpalm demonstrated most of the classic symptoms of CTS, including diminished sensitivity and thenar atrophy (4). Brown 
and Coulson (5) reported two cases where patients had triggering at the transverse carpal ligament secondary to FTS, as well as paresthesia in the area innervated by the median nerve. In all of these patients, including the one described in this case report, local excision, along with carpal tunnel decompression, promptly relieved the symptoms.

The texture of the tumours reported in the literature was consistently described as being elastic, firm or hard. The mean size of tumours described was about $2 \mathrm{~cm}$ and usually grey-white in colour. The mean age of FTS formation is about 36 years, and is generally more common in males than females, with the former representing $75 \%$ of the Chung and Enzinger study (2).

Macroscopically, all the tumours reviewed in the literature were reported to be well circumscribed, clearly delineated and sometime encapsulated (2). All cases of FTS describe spindle-shaped fibroblasts embedded in a dense, fibrous 'hyaline' (eosinophilic) stroma abundant in collagen (6). Within the dense stroma are often scattered thin-

\section{REFERENCES}

1. Geschickter CF, Copeland MM. Tumours of Bone, 3rd edn. Philadelphia: JB Lippincott, 1949:693-5.

2. Chung EB, Enzinger FM. Fibroma of tendon sheath. Cancer 1979;44:1945-54.

3. Sarma D, Weilbaecher TG, Rodriguez FH. Fibroma of tendon sheath. J Surg Oncol 1986;32:230-2.

4. Evangelisti S, Reale VF. Fibroma of tendon sheath as a cause of carpal tunnel syndrome. J Hand Surg 1992;17A:1026-7. walled, small blood vessels penetrating random slit-like channels (7).

Many characteristics of the tumour described in this report correlate with those described in the literature.

In most of the reported cases, the tumours were well circumscribed and multilobulated (8). In all cases, the tumours were attached, or closely related, to tendons or tendon sheaths, but were readily removed at surgery. This was not so with the present case, where the common digital artery of the middle and ring fingers required resection because it was encased within the substance of the tumour. In addition, the motor branches of the lumbricals of the middle and ring fingers had to be sacrificed for the same reason, which indicates that this histologically benign tumour may, on occasion, behave aggressively in a localized area.

Although originally this case was thought to be a possible schwannoma, a nerve sheath tumour or an angiofibroma, it is now evident it represents the rare entity of FTS as a cause of CTS.

5. Brown LP, Coulson DB. Triggering at the carpal tunnel with incipient carpal tunnel syndrome. J Bone Joint Surg 1974;56A:623-4.

6. Cooper PH. Fibroma of tendon sheath. J Am Acad Dermatol 1984;11:625-8.

7. Hashimoto H, Tsuneyoshi M, Daimaru Y. Fibroma of tendon sheath: A tumor of myofibroblasts. Acta Pathol Jpn 1985;35:1099-107.

8. Jablokow VR, Kathuria S. Fibroma of tendon sheath. J Surg Oncol 1982;90-2. 